\section{Cahiers de Narratologie}

Analyse et théorie narratives

11 | 2004

Figures de la lecture et du lecteur

\title{
Programmation et représentation dans la fabula du désir du lecteur
}

\section{Emmanuelle Occelli}

\section{(2) OpenEdition}

Journals

Édition électronique

URL : http://journals.openedition.org/narratologie/14

DOI : 10.4000/narratologie. 14

ISSN : 1765-307X

Éditeur

LIRCES

Référence électronique

Emmanuelle Occelli, «Programmation et représentation dans la fabula du désir du lecteur », Cahiers de Narratologie [En ligne], 11 | 2004, mis en ligne le 01 janvier 2004, consulté le 15 novembre 2019. URL : http://journals.openedition.org/narratologie/14 ; DOI : 10.4000/narratologie.14

Ce document a été généré automatiquement le 15 novembre 2019.

\section{(c) (i) (9)}

Cahiers de Narratologie - Analyse et théorie narratives est mis à disposition selon les termes de la licence Creative Commons Attribution - Pas d'Utilisation Commerciale - Pas de Modification 4.0 International. 


\title{
Programmation et représentation dans la fabula du désir du lecteur
}

\author{
Emmanuelle Occelli
}

1 Le crime d'olga Arbélina ${ }^{1}$ est le cinquième roman d'Andreï Makine, publié en 1998, trois ans après Le testament français qui lui a valu le prix Goncourt, le prix Médicis et le prix Goncourt des lycéens, mais le roman a été commencé un an avant, inspiré d'une anecdote qu'on lui a racontée. « Une princesse russe vivait dans un village d'immigrés. Les gens pensaient qu'entre elle et son enfant, il y avait... et, à ce moment du récit, les gens se taisaient. J'ai pensé qu'il y avait là, confie Makine ${ }^{2}$ une matière poétique forte, une situation qui dépassait même celle des romans de Dostoïevski, dont les héros s'arrêtent à la frontière. Chez Olga, cette frontière tombe et elle doit vivre au-delà de cette limite. " Celle-ci étant, on l'aura compris, l'inceste. L'auteur ajoute : « Le crime d'olga Arbélina est un roman très pudique mais, à Lyon, des lectrices ont jeté le livre à la tête du libraire."

Olga Arbélina n'est d'abord qu'un nom tracé sur une pierre tombale, celui d'une princesse morte dans les années soixante et sur laquelle le gardien du cimetière russe, à Paris, s'avère intarissable lorsqu'un écrivain russe exilé, d'une quarantaine d'années, s'attarde devant la tombe après que le groupe de visiteurs a depuis longtemps quitté les lieux. Le gardien entreprend le récit de la vie d'olga dont il semble ne rien ignorer.

Olga est alors cette femme de quarante-sept ans que, par une journée de juillet 1947, les habitants de Villiers-la-Forêt, une petite ville proche de Paris, découvrent presque nue, choquée, à côté d'un homme mort, Serge Goletz, ancien officier de l'Armée blanche, sur les berges d'une rivière serpentant à travers la forêt où Olga a sa maison. Les villersois la connaissent. Olga s'est installée avec son enfant hémophile dans cette ville de province juste avant la guerre, au printemps 1939, dans une maison isolée, à l'arrière d'une ancienne fabrique de bière transformée en appartements pour les réfugiés russes. Goletz et Olga ne se fréquentaient guère, à la connaissance des autochtones : comment expliquer leur promenade en barque et le chavirement? Tous s'improvisent détectives comme dans les romans policiers. Qu'en est-il de son amant parisien, un journaliste 
connu et marié, qu'Olga voyait régulièrement? Ménage à trois? Mais l'enquête conclut à un accident et prononce un non-lieu.

4 La nuit tombe et le gardien fait entrer l'écrivain russe, narrataire de son récit, dans sa maison, avant de poursuivre en remontant au 25 août 1946, date à laquelle Olga se fait avorter clandestinement chez Li, une amie d'enfance qui réside à Paris où elle exerce la profession de photographe. Dans sa cave, elle fait poser ses clients derrière des panneaux en trompe-l'œil figurant des personnages mythologiques, historiques et littéraires aux visages évidés. De retour chez elle, Olga reprend ses activités de bibliothécaire, la vie paisible qu'elle partage avec son enfant malade déjà adolescent, tout en réfléchissant au temps qui passe et en se remémorant son passé : son époux qui l'a quittée lorsque leur enfant n'avait que six ans, son enfance à Ostrov dans la maison nobiliaire où se donnaient des bals costumés, l'interminable révolution bolchevique, son exil en même temps que Li, son arrivée à Paris en 1922, la naissance de son fils en 1932, son installation au sein de la communauté russe vieillissante et atone comme un matin de neige. Dans cette ville de province de l'après-guerre français où seules les variations du ciel et des saisons apportent l'improbable, Olga vit hantée par le caractère irrémédiable de sa vie. Solitaire, renfermée, elle ira jusqu'à connaître la folie la plus sombre, jusqu'à devenir étrangère à elle-même et à son propre fils. Dans cette lente observation, entrecoupée d'événements blessants, d'un temps qui passe mais ne passe pas et jamais ne s'accomplit, surgit la figure de Goletz qui soigne son fils quand son genou enfle à la moindre écorchure, sensible aux charmes d'olga, trop entreprenant à son goût, sachant sur elle des informations compromettantes. L'accident en barque comble Olga au point qu'elle s'en accuse dans un geste de folie désespérée. Tandis qu'elle est recluse dans une maison de repos, son mari vient lui annoncer qu'il retourne en U.R.S.S. en emmenant leur fils, sensible aux promesses de clémence de Staline pour les émigrés repentis, comme Li d'ailleurs en a elle-même l'intention. Olga reste des années sans nouvelles jusqu'à ce qu'un homme revenant de Russie lui apprenne que son mari et Li ont péri en camp de rééducation. Cet individu, avec qui Olga partagera la fin de son existence, sans savoir ce qu'est devenu son fils, sera son confident.

5 Au terme de son récit, le gardien reconduit l'écrivain russe exilé au portail du cimetière.

6 Le crime d'Olga Arbélina d'Andreï Makine présente la particularité de solliciter une intense activité de la part du lecteur, perpétuellement dérouté tout en étant conforté dans ses attentes. A la fois «texte de plaisir » et «texte de jouissance » pour reprendre la fameuse distinction de Roland Barthes ${ }^{3}$. La lecture est à la fois confortable psychologiquement et déstabilisatrice culturellement, historiquement, sur le plan axiologique. Cela tient à l'élaboration d'un dispositif textuel complexe qui programme efficacement le désir de son lecteur par un ensemble de procédés convergents. Nous employons ce terme de «lecteur» dans l'acception que lui donne Vincent Jouve. Il ne s'agit pas, bien entendu, du lecteur réel, physique, particulier, qui peut ne pas accepter le rôle que lui assigne le texte. En témoignent ces lectrices lyonnaises qui ont jeté le livre à la tête du libraire mais, même dans ce cas, les réactions de ces lectrices individuelles sont déterminées par la position du lecteur virtuel. Le lecteur virtuel ou implicite correspond « à la somme des instructions du roman sur la façon dont il doit être lu $»^{4}$. Jouve postule néanmoins une corrélation entre «le destinataire implicite des effets de lecture programmés par le texte » et les réactions du lecteur réel, «impliqué dans sa lecture avec son intelligence et son inconscient $»^{5}$, dans la mesure où le rôle 
proposé au lecteur réel constitue un point de passage obligé dans sa relation au texte, qu'il le trouve à son goût ou non.

7 Globalement, ces procédés programmatifs sont de deux sortes dans Le crime d'olga Arbélina. Tout d'abord, le titre de l'œuvre. Sans aller jusqu'à «l'épanode narrative » qu'Alain Tassel définit comme une "germination des mots du titre » dans le corps du texte ${ }^{6}$, « chaque unité lexicale fécond(ant) un développement narratif ou discursif $»^{7}$, le titre est néanmoins essaimé plus ou moins fidèlement dans le texte, métaphorisé, voire invalidé (quand Olga s'accuse du crime de Goletz alors qu'il s'agit d'un accident dans les faits), conviant ainsi le lecteur à une enquête comme les Villersois après la découverte des deux corps sur la berge.

Parallèlement à « la réverbération du titre sur la trame textuelle » ${ }^{8}$, la représentation du désir d'olga préfigure celui du lecteur, lui désigne l'attitude à adopter, tout en entretenant l'illusion que le personnage est constamment en retard sur ses attentes. Le parcours de ce dernier balise celui du lecteur qui a pourtant l'impression que le comportement d'olga reflète, en différé, le sien. C'est moins une mise en abyme du désir métadiégétique du lecteur qu'un dispositif destiné à le susciter ; la représentation du désir intradiégétique est, en effet, programmative plutôt que reproductrice.

Pour ce qui est du titre, il joue un rôle fondamental dans la construction d'un horizon d'attente en permettant au lecteur de circonscrire le contenu du livre dans un champ de possibles et, comme tel, le titre participe d'un ensemble d'activités lectorales appelé «topic ». Le topic, selon Umberto Eco, est un phénomène pragmatique qui fixe les limites d'un texte' ${ }^{9}$. Il est, en effet, courant de penser que le titre d'un morceau en fixe le thème. Or, dans Le crime d'olga Arbélina, le crime d'olga (sa complaisance envers les relations incestueuses que son fils entretient avec elle pendant qu'il la croit endormie) n'est qu'instrumental par rapport au propos principal. Makine a donné un titre trompeur parce qu'il savait que le lecteur utiliserait le titre comme indicateur thématique. Le topic reste alors à chercher. Le lecteur, dans ce roman, doit suspecter quelque chose dans la dispositio. Le crime d'olga oriente le lecteur vers le topic véritable par analogie (désir tératologique) et passage de l'individuel au collectif (le fils hémophile $=$ les communistes $/$ la mère faisant semblant de ne rien voir $=$ la scotomisation que suscitent généralement les idéologies totalitaires). Le véritable topic est inséré au cœur du roman et ne fait l'objet que de quelques pages : la représentation de la Révolution bolchevique. La lecture n'est donc pas linéaire. Arrivé au centre du livre, le lecteur est contraint de regarder en arrière et, parvenu au terme du roman, il lui faut le relire car la fin articule la représentation de l'Histoire à une problématique identitaire. Cette œuvre comporte plusieurs topics dont on modifie la hiérarchie de manière rétrospective avec l'explicit ${ }^{10}$. La détermination du topic véritable dans $L e$ crime d'Olga Arbélina nécessite un mouvement coopératif (pragmatique) qui amène le lecteur à inférer que le crime d'olga entretient une relation allégorique ou métaphorique avec la Révolution bolchevique.

Dès les pages 102-103, le lecteur se pose une question sur la signification du geste du fils surpris par Olga : pourquoi met-il une poudre dans l'infusion du soir de sa mère ? Le lecteur formule une double hypothèse que le texte ne cherche pas à désambiguïser tout de suite: soit le fils empoisonne progressivement sa mère, soit il lui administre un puissant narcotique. Une fois la seconde hypothèse avérée, la question du pourquoi reste longtemps sans réponse, du moins sans réponse formulée par la mère : « (...) un texte narratif introduit des signaux textuels de différents types pour souligner que la 
disjonction qui va être occurrente est importante. Appelons-les signaux de suspense. Ils peuvent, par exemple, consister à différer la réponse à la question implicite du lecteur $»^{11}$. Repérons ces signaux de suspense. L'auteur s'emploie à nous signaler l'état d'interrogation, d'attente du personnage d'olga, état qui représente celui du lecteur et le fonde. En décrivant l'attente du personnage, l'auteur joue avec le désir du lecteur. Dès la page 90, un autre épisode installe progressivement l'attente dans la fabula. Olga, qui avait emprunté à son amie $\mathrm{Li}$, un appareil photographique se déclenchant automatiquement au changement de la lumière (cinq poses à trois secondes d'intervalle) l'oublie sur une étagère dans sa chambre jusqu'au jour où elle le rend à Li. Cette dernière s'aperçoit que l'appareil s'est déclenché et lui envoie trois clichés, les deux premiers n'étant pas réussis faute d'une lumière suffisante. Le premier représente le chat à l'affût alors qu'il dort habituellement à la cuisine. Sur le deuxième, olga est de dos, assise sur le bord de son lit ôtant sa chemise de nuit: la nuit de septembre était chaude. Sur le dernier, Olga nue ouvre la fenêtre. Olga contemple les photographies. La narration en focalisation interne signale l'impossibilité de dire, souligne un non-dit. Cet interdit crée aussitôt un désir chez le lecteur et stimule sa libido sciendi. Le processus est amorcé :

«Et encore une singularité qu'elle ne parvenait pas à formuler, un secret qui dépassait les mots et dont le goût, comme celui de la menthe, glaçait l'odorat, soulevait la poitrine...»

«Pendant tout le temps qu'elle examinait les clichés, la voix de la "petite garce » ne cessa de souligner des incohérences étranges : « Pourquoi les deux premiers sont tout noirs, le troisième à peine éclairé et les deux derniers réussis ? - Tais-toi, c'est sans doute la faute de l'appareil... - Et pourquoi la porte est ouverte ? - Un courant d'air. - Et le chat ? - Tais-toi, je ne veux rien savoir ! » (p.90)

11 Le dédoublement du personnage d'olga par une voix intérieure qui énonce explicitement les questions que le lecteur se pose rend manifeste que les structures discursives travaillent à préparer les attentes du lecteur. Par ce dédoublement du personnage, la narration devient dialogique et inscrit le lecteur dans la fabula en lui assignant un rôle : pousser le personnage dans ses retranchements pour lui faire dire ce qu'il désire entendre :

«Toutes ces photographies, c'est bien joli, mais il vaudrait mieux penser de temps en temps à ton fils..."

«Olga ne répondit pas. (...) Que pouvait-elle répondre à la voix qui la poursuivait de ses reproches? (...) Répondre qu'il lui arrivait de faire des songes où l'évolution de la maladie s'accélérait et s'achevait en une seule journée. (...) La petite garce se taisai $t » .(p p .90-91)$

$12 \mathrm{Au}$ lieu de répondre à la question du lecteur, la narration introduit de nouveaux épisodes. Olga se rend à la bibliothèque où elle travaille en emportant avec elle les clichés pour les examiner à nouveau :

«Il y avait dans son désir une part de cette attirance maniaque que provoquent certains clichés vers lesquels le regard est entraîné avec la dépendance d'un morphinomane, comme pour s'assurer que leur charme mystérieux ne s'est pas effacé ou, au contraire, en espérant y découvrir un nouveau détail qui transformera leur monde instantané ». (p.93, nous soulignons)

13 En signalant le désir du personnage, la narration pointe métatextuellement celui du lecteur. Mais le récit diffère encore la réponse car les tâches de bibliothécaire d'olga l'accaparent. Ce n'est que plus tard que celle-ci les examine à nouveau :

«Plus intensément encore que la veille, cette femme surprise devant la fenêtre noire donnait l'impression de vaciller au bord d'une étonnante révélation. « Elle est 
totalement ... comment dire ? méconnaissable ? autre ? Enfin, moi, j'étais autre à cet instant... » Elle inclinait le cliché pour changer l'angle d'éclairage en espérant que les mots allaient se détacher soudain de sa surface et condenser son mystère dans une formule... Les premiers lecteurs du jour apparaissaient dans la porte ». (pp. 94-95) de « révélation » alimente le suspense. Plusieurs jours s'écoulent, la narration digresse d'abord puis nous livre une révélation :

« $\mathrm{Au}$ cours d'une soirée claire et fraîche de l'arrière-saison, dans un moment de grande sérénité, elle surprendrait son fils près de ce petit récipient en cuivre dans lequel se décantait son infusion de fleurs de houblon. Elle l'apercevrait figé dans cette attente brève et crispée qui suit un geste qu'on veut à tout prix secret. Oui, cette fixité hypnotique qui s'intercale entre ce geste dangereux ou criminel et la décontraction exagérée des mouvements et des paroles qui viennent après. $\mathrm{Ce}$ qu'elle croirait alors deviner lui paraîtrait alors d'une monstruosité si invraisemblable qu'instinctivement elle reculerait de quelques pas. Comme si elle avait désiré remonter le temps, en pressentant déjà que le retour à leur vie d'autrefois devenait, à cet instant là-même, impossible». (pp.102-103, nous soulignons)

Coup de théâtre! Non seulement la réponse attendue aux photographies n'est pas apportée mais une autre question bien plus cruciale est désormais posée. L'adjectif "criminel» pour caractériser le geste du fils fait fonction de marqueur de topic puisqu'il est déjà présent dans le titre du roman sous une forme substantivée. L'effet pragmatique est fort et relance la coopération interprétative du lecteur. En effet, dans le titre, le crime est celui d'olga. Comment le geste filial « criminel » a-t-il pu justifier cette attribution maternelle? Comment le transfert de criminalité s'est-il opéré ? Le chapitre se clôt sur ces signaux de suspense et fait coïncider la fin du chapitre avec une situation de disjonction que la narration souligne par l'emploi du mot « désir » dans la comparaison finale. Le désir du personnage de revenir en arrière rappelle au lecteur son désir de voir formuler une réponse concernant les trois clichés. A la question: « Pourquoi l'appareil, sensible à la lumière, s'est-il brusquement déclenché dans la nuit, pendant qu'olga dormait? » s'en substitue une autre : «Pourquoi le fils d'olga ajoute-til une poudre à l'infusion de sa mère à son insu? » Mais la narration retarde la réponse. Le geste criminel du fils sur lequel se clôt le chapitre correspond à un effet d'annonce : le conditionnel (futur du passé) souligne la valeur proleptique de la narration et le chapitre suivant décrit les circonstances dans lesquelles olga a vu son fils agir sans qu'il sans doute.

La scène en focalisation interne joue sur l'instance lectorale que Vincent Jouve appelle le $l u^{12}$. Olga jardine devant la maison, au crépuscule, plongée dans ses pensées :

"C'est dans cette distraction bienheureuse qu'elle poursuivait son travail. Penchée vers le sol, elle parvint enfin sous la fenêtre de la cuisine et se redressa.

Trop brusquement ! Le vertige l'assourdit et fit tanguer la fenêtre éclairée et les rideaux. Son corps se remplit d'une faiblesse mate, vaporeuse. Le mur sur lequel elle appuya sa paume sembla se ployer doucement. Pour arrêter ce flottement, elle immobilisa le regard sur l'écart lumineux entre les rideaux... Elle vit un inconnu, un très jeune homme qui se tenait près du fourneau...

Elle vit son geste. Avec cette précision qu'ont les mouvements et les objets derrière la fenêtre d'une pièce observée d'un extérieur nocturne, par un temps froid. Une précision presque hallucinante à cause du vertige ». (p.105) 
17 Le noir dans lequel se situe l'observateur, son extériorité par rapport au sujet observé, sa situation derrière la fenêtre, protégé par le rideau qui le masque : voir sans être vu. La narration exploite le fantasme du voyeur qui sommeille en chaque lecteur, le livre étant cette fenêtre lui découvrant les faits et gestes de personnages, parfois leur intimité, tout en maintenant le lecteur dans la position confortable du voyeur qui s'abrite derrière son extériorité au lieu observé. D’après Michel Picard, le lecteur ne s'identifie pas à un personnage mais à un personnage en situation. Cette appropriation a une base libidinale, ici le voyeurisme, et conduit à « intégrer temporairement comme pour les essayer, des situations dont les héros ont seulement pour fonction de dessiner les contours $»^{13}$. Cette souplesse dans l'identification relève du jeu (playing, par opposition à game). Michel Picard cite Philippe Gutton, page 112 : « Le jeu est un « faire comme si ». (...) Le jeu suppose un désengagement par rapport à la situation, de telle sorte qu'au moment où celle-ci est jouée, elle se trouve, en quelque sorte, absente ${ }^{14}$. Le lecteur se dédouble en sujet joué s'abandonnant aux identifications selon le principe de plaisir et en sujet jouant qui se situe dans le réel. La scène mettant Olga en situation de voir de l'extérieur ce que son fils fait à l'intérieur de la maison met en abyme le dédoublement de l'instance lectorale à la fois dedans et dehors, jouée et jouant. La narration, toujours en focalisation interne, fait alors pénétrer le lecteur dans la conscience d'olga et à travers les réactions qui lui sont livrées, ce sont les siennes que désigne le texte :

« Devinant tout, ne comprenant encore rien, elle vit sous ses paupières s'assembler des fragments dispersés : ces doigts en voltige au-dessus des fleurs de houblon infusées, les trois photographies de la femme nue, la porte ouverte la nuit où elles avaient été prises, deux jours passés chez Li, l'avortement...Ses yeux noyés dans l'épaisseur cotonneuse du vertige discernaient déjà avec horreur le sens de cette mosaïque désassemblée. Mais la pensée, engourdie par la montée du sang, se taisait ». ( p.106)

18 La métaphore de la mosaïque assigne au lecteur une tâche interprétative : maintenant en possession de toutes les pièces du puzzle, il doit les assembler pour qu'elles fassent sens. En réalité, il manque une pièce que le texte s'empresse de fournir en exhibant la règle de coréférence qui sert à comprendre les expressions déictiques ou anaphoriques. Cet élément manquant est celui que la narration ne cesse d'évoquer sans qu'il n'ait jamais été le thème d'une description :

«Son regard était à présent fixé sur le jeune inconnu qui, dans la cuisine éclairée,

feuilletait nonchalamment un cahier ouvert sur la table. C'était son fils ! »(p.107)

D'après les mentions précédentes du fils, il est impossible au lecteur de faire le lien entre l'anaphore : « le jeune inconnu » et le fils d'Olga. Excepté le soir où Olga a surpris le "geste criminel » de son fils (et ce dernier n'était pas décrit, c'étaient les réactions de la mère qui étaient en jeu), le lecteur n'a jusqu'à présent jamais eu accès directement au personnage filial. C'est uniquement au travers des préoccupations maternelles, le récit étant en focalisation interne la plupart du temps, qu'il détient des informations sur lui. Or le comportement d'olga laissait supposer que son fils était un enfant de sept ans: elle reprise ses vêtements lorsqu'il fait ses devoirs, elle prépare ses affaires pendant qu'il dort pour le lendemain matin (p.102). La première fois que le texte le désigne, c'est sous le vocable "d'enfant » et il est présenté comme dormant dans sa chambre d'un sommeil calme et pur (p.78). La seconde fois, c'est indirectement qu'il est évoqué. Le narrateur omniscient relaie le point de vue interne. Olga découvre les trois clichés et pense à son fils. Du seuil de sa chambre, elle tend l'oreille pour savoir s'il 
dort. Le narrateur apporte alors au lecteur quelques informations sur la disposition des pièces: " la chambre de l'enfant ", la bibliothèque que l'enfant appelle "la pièce aux livres »(p.91). Puis la focalisation interne permet d'épouser les pensées d'olga au sujet de son fils, présenté comme un enfant malade, condamné et incapable d'accéder au rayonnage le plus élevé de la bibliothèque sur lequel sont rangés des ouvrages sur son hémophilie. Entre cette présentation et la vision d'un jeune homme de quatorze ans, le lecteur ne peut établir de lien qu'au prix d'un réajustement stéréoscopique faisant coïncider les deux images. Olga n'a pas vu grandir son fils : le temps s'est arrêté pour elle lorsque la seconde guerre mondiale a commencé. On est maintenant en 1946 :

«Elle comprenait maintenant que le jeune homme avait surgi au moment du vertige, le visage et le corps mûris par l'horreur de la mosaïque qui avait révélé l'impensable. Oui, ce très jeune homme mince, pâle, avec le reflet transparent, presque invisible de la toute première moustache, appartenait au monde de la mosaïque qui, au contact de la pensée, se transformait en un reptile luisant, aux yeux vitreux, indéchiffrables. (pp.107-108)»

Le motif du reptile qui émerge des pièces du puzzle commençant à s'assembler fait cette fois appel à une autre compétence du lecteur: sa connaissance des scénarios intertextuels. Avec la règle de coréférence, le lecteur se souvient qu'Olga en visite chez Li pour qu'elle l'avorte a décrit les panneaux en trompe-l'œil que celle-ci utilise pour photographier ses clients. L'un d'eux représente Psyché et Cupidon. Or le serpent intervient dans ce mythe pour désigner Cupidon qui rejoint Psyché toutes les nuits sans qu'elle puisse le voir. Les indices laissés par l'auteur à l'attention du lecteur doivent susciter son activité interprétative, car la formulation de la réponse désirée, à la question que se pose ce dernier, via Olga, est retardée par une pirouette : Olga boit la tisane dans laquelle elle a vu son fils jeter une mystérieuse poudre pour mettre fin aux pensées qui la tourmentent:

«La mosaïque s'effaça. Le reptile dans son cerveau creva sans bruit, en enfonçant sous ses paupières une multitude d'aiguilles rouges. La cuisine reprenait ses dimensions habituelles » (p.111)

21 Le scénario fantastique amorcé s'enraye aussitôt. En effet, toujours sous le choc de la vision et de la prise de conscience de l'âge de son fils qui grandit à ses yeux de sept années d'un coup, les pensées d'olga se mettent à lui dessiner un univers hallucinant où la familiarité des lieux côtoie l'étrange :

« Elle appuya sur l'interrupteur et réprima un « ah » en portant sa main à ses lèvres tant l'intérieur de la cuisine lui semblait rapetissé. La taille du jeune homme, même invisible, s'imposait à ces murs, aux meubles, en les réduisant comme ces rêves pénibles qui nous font pénétrer dans un appartement familier qui se resserre à vue d'œil et imite à la fin l'habitacle des figurines d'une boîte à musique...Oui, en s'arrêtant au milieu de la cuisine, elle avait l'impression d'examiner l'intérieur d'une maison de poupée dont la petitesse, à la fois séduisante et dénaturée, dégageait une menace obscure. Même la petite casserole sur le fourneau paraissait plus petite qu'avant et révélait enfin sa vraie forme - légèrement évasée, ventrue ». (p.110)

Nul doute que l'auteur s'amuse à tester la connaissance des scénarios intertextuels de son lecteur qui aura reconnu Alice au pays des merveilles de Lewis Caroll dans cette vision déformée par la peur d'olga. Sous l'effet de l'infusion, Olga s'endort mais, au matin, des cognements à la porte d'entrée la réveillent et la question ressurgit :

«Et s'ils ont deviné? se demanda-t-elle, perplexe. Mais deviner quoi? " Absurdement, elle imagina que les autres pouvaient découvrir qu'elle leur avait caché l'âge de son fils. (pp.114-115) 

comme avec la visite d'une infirmière :

«Elle a flairé quelque chose», pensa de nouveau Olga, et elle se rabroua immédiatement : « Idiote ! Qu'y a-t-il à flairer dans cette masure ?» (pp.116-117)

Puis des épisodes narratifs suspendent encore la réponse. Mais le soir, alors qu'olga se coiffe devant le miroir de sa chambre, elle voit le reflet de la porte qui s'ouvre :

«Cet entrebâillement silencieux qui laissa entrer le souffle d'une fenêtre ouverte créa une étrange attente. Olga se souvint de la nuit où un courant d'air l'avait réveillée en faisant grincer la porte de sa chambre, oui, la nuit des trois clichés pris par l'appareil-livre. D'aussi loin qu'elle se rappelât, cette porte grinçait légèrement (il y a toujours, dans une maison, un couteau qui coupe mieux que les autres et une chaise qu'on évite de donner aux invités) ». (pp.121-122, nous soulignons)

Le mot «attente » vient rappeler que la réponse que désire le lecteur est toujours en suspens. En même temps l'auteur joue avec la connaissance des scénarios communs du lecteur en rapprochant le grincement de la porte des événements qu'on rencontre fréquemment dans la vie quotidienne et des habitudes d'une maisonnée. Mais c'est la connaissance des scénarios intertextuels qui est requise ici. Le récit prend un tour policier avec les indices qu'Olga s'emploie à relever: des traces récentes d'huile se trouvent sur les gonds de la porte. Le chapitre se clôt ironiquement sur un signal de suspense :

«Il lui fallut un effort musculaire pour chasser de son visage ce sourire tendu cette joie d'avoir rejeté, ou au moins retardé la conclusion définitive... » (p.123)

Si Olga a trouvé la réponse, elle ne la formule pas pour autant, laissant le lecteur insatisfait. La scène de l'infusion se reproduit (p.133) sans que l'explication escomptée ne soit apportée. Cependant l'hypothèse de l'empoisonnement est maintenant écartée avec la répétition du geste et l'absence de symptômes, ce que souligne l'ultime phrase du chapitre suivant :

« Mais surtout, cette fois, il n'y eut plus de doute ». (p.134)

L'interrogation du lecteur porte désormais sur la raison qui pousse le fils à administrer une drogue soporifique à sa mère. La scène se réitère mentionnant qu'olga a compris sa signification mais ne peut la verbaliser (p.137). Au lieu des mots, s'impose l'image du reptile. Cherchant à expliquer le geste filial, olga se remémore son passé avec le père de l'enfant parti depuis plus de sept ans. Le suspense est relancé lorsqu'elle s'aperçoit qu'elle ne dort toujours pas à minuit passé alors qu'elle a surpris le même geste du jeune homme et qu'elle a bu la tisane. La narration court le risque de décevoir le lecteur. En effet, Olga réinterprète la scène en la minimisant :

«Le geste bizarre et suspect de l'adolescent dans la cuisine? Rien d'autre qu'un de ces gestes farfelus, souvent maniaques, de celui qui se croit seul dans la pièce ». (p.

145)

Tous ses soupçons et par là-même ceux du lecteur, sont renvoyés à un délire de femme seule. La seconde partie aurait pu s'achever ainsi, de façon déceptive pour le lecteur sans que rien ne fût dit. Mais le dernier paragraphe au conditionnel, à valeur de futur du passé, se fait proleptique et relance le questionnement :

«Très peu de jours après cette nuit blanche qui sembla dissiper l'étouffement des doutes, Olga devinerait pourquoi, ce soir de novembre, les somnifères n'avaient pas eu d'effet. Elle comprendrait que la poudre que l'adolescent déversait dans l'infusion n'avait pas eu le temps de se dissoudre et que, pressée de démentir son 
horrible intuition, elle avait avalé le liquide sans l'avoir brassé...Elle comprendrait tout ». (p.146) souvenirs d'olga en Russie de 1906 jusqu'à la Révolution bolchevique, puis son immigration en France à la suite de cette tourmente en 1922 et son installation en 1939 dans la fabrique en banlieue parisienne. Cette représentation de l'Histoire semble, à première lecture, sans rapport avec la question posée : pourquoi le fils d'olga la droguet-il certains soirs? La solution de continuité entre les deux séquences reproduit la contiguïté des images qui peuplent les rêves des dormeurs. Or, c'est précisément le sommeil artificiel de la mère qui suscite les interrogations lectorales. Mais le retour à la vie quotidienne dans la fabrique transformée en appartements, sans apporter de réponse, se fait plus explicite. Cette fois, avant de perdre conscience, olga a le temps d'apercevoir son fils pénétrer dans sa chambre. Au matin, les rideaux qui étaient décrochés la veille sont ajustés sur la tringle, attestant de la présence nocturne filiale qui reste pourtant dans le non-dit:

«Cela était le soir de la veille, la nuit. Elle le comprenait mais parvenait encore à le garder innommé : cela. » (p.185)

31 Le soir, en rentrant, Olga formule enfin les mots que le lecteur attend depuis plus de cent pages :

«Il était dans mon corps...» Elle comprenait maintenant pourquoi cela restait innommé. Car pour nommer, il ne fallait pas parler de sentiments, mais dire ces mots rudes, laids, frustes qui se transvasaient en coulée poisseuse, dans sa gorge : « Il me violait. Il me possédait quand il en avait envie. Il me déshabillait, me prenait, me rhabillait...» (p.191)

Mais la réponse à la question de la signification du geste criminel du fils a fait surgir une autre interrogation: la représentation de l'Histoire de la Russie de 1906 à 1918 environ n'est-elle qu'un procédé dilatoire ou a-t-elle un lien avec le désir incestueux du fils ? La narration met en relation la naissance de l'enfant et les bouleversements politiques :

L'enfant naît en 1932, l'année où l'émigrant russe Pavel Gorgoulov tue d'un coup de revolver le président Paul Doumer. Les Russes se transmettent les dernières paroles du condamné traîné vers l'échafaud: "Le monde doit être gouverné par la Troïka verte ! » On dit qu'il est devenu fou bien avant son crime. Oui, la même année : il est difficile de ne pas penser au fracas du couperet et au jaillissement du sang. Elle y pense en apprenant l'hémophilie de l'enfant (une minuscule écorchure survenue pendant l'accouchement laisse serpenter un filet de sang interminable). (p.171)

Quoi qu'il en soit, après avoir nommé la raison pour laquelle son fils la droguait, Olga jette désormais le breuvage lorsqu'elle y aperçoit un reflet de poudre blanche et fait semblant de dormir quand l'adolescent la rejoint dans sa chambre. Désespérée, elle se rend chez Li pour tout lui avouer mais commence par évoquer les impressions qu'elle a ressenties lors de la Révolution bolchevique :

«L'impression qu'un jour la vie avait perdu une certaine rectitude, justesse, régularité. Un jour où s'était introduit dans leur vie russe, dans le pays tout entier, un étrange caprice. Soudain l'envie les avait pris de prouver que cette rectitude n'était qu'une chimère, un préjugé de boutiquiers. Et qu'on pouvait vivre sans la respecter ou mieux encore en la narguant. De plus, la vie semblait leur donner 
raison : un paysan sibérien nommait et chassait les ministres, " purifiait » comme il appelait ces accouplements, les dames d'honneur de la tsarine et, selon les mauvaises langues, la tsarine elle-même, toutes subjuguées par sa force charnelle ». (p.205, nous soulignons)

Pour être correctement interprété, le passage requiert la compétence idéologique du lecteur afin d'expliciter ce qui est suggéré. L'axiologie sous-jacente établit une équivalence entre le tsarisme et la droiture, l'ordre, l'équilibre social, jusqu'à Raspoutine. Après la chute du tsarisme, avec les bolcheviks, l'inversion des valeurs et la transgression régnèrent :

«Tuer un policier devenait un exploit au nom de la liberté... » (p.205)

Un texte remarquable de Pierre Pascal ${ }^{15}$ atteste de l'inversion des valeurs inhérente à la révolution bolchevique. Pierre Pascal est l'un des premiers témoins étrangers de la révolution russe à avoir tenu registre de ce qu'il a vu depuis 1917 jusqu'à 1927. Il appartient à une génération de jeunes Français passés par l'Ecole normale supérieure (promotion 1910), étudiant en lettres et slavophile. Le bolchevisme de Pascal, fervent catholique, est un messianisme communautaire proche de la morale fraternitaire annoncée par le Christ. Témoin cet extrait de son journal, cité par le grand historien de l'idéologie communiste, François Furet ${ }^{16}$, qui veut décrire la Russie de la révolution et brosse le tableau d'un monde déréglé par une apocalypse égalitaire :

«Spectacle unique et enivrant : la démolition d'une société. C'est maintenant que se réalise le quatrième psaume des vêpres du dimanche et le "Magnificat »: les puissants renversés de leur trône et le pauvre relevé de sa crotte. Les maîtres de maison sont confinés dans une pièce, et dans chaque autre pièce est logée une famille. Il n'y a plus de riches: simplement des pauvres et des plus pauvres. Le savoir ne confère plus ni privilège ni respect. L'ex-ouvrier promu directeur commande les ingénieurs. Les salaires, du haut et du bas, se rapprochent. Le droit de propriété est réduit aux hardes personnelles. Le juge n'est plus tenu d'appliquer la loi, lorsque son sens de l'équité prolétarienne la contredit. Le mariage n'est plus qu'une inscription à l'état civil, et le divorce peut être signifié par carte postale. Les enfants sont instruits à surveiller les parents. Les sentiments de générosité sont chassés par le malheur des temps : on se compte en famille les bouchées de pain ou les grammes de sucre. La douceur est réputée vice. La pitié a été tuée par l'omniprésence de la mort. L'amitié ne subsiste que comme camaraderie ». (p.16)

En utilisant la capacité de repérer les sélections contextuelles et circonstancielles, le lecteur peut inférer que la naissance du fils d'olga est marquée du sceau de la perversion, du renversement des valeurs qui caractérisent l'avènement au pouvoir des bolcheviks et que symbolise le portrait de la grand-mère d'olga, une comtesse aux idées conservatrices, suspendu «tête en bas » (p.205). En effet, on se souvient que son fils est né l'année où le président Doumer, représentant l'ordre, au même titre que le policier, fut assassiné par un émigrant russe contaminé par ce dérèglement attaché à l'instauration du régime bolchevique. Les désirs incestueux du fils hémophile sont ainsi présentés comme la manifestation des valeurs perverties des insurgés communistes, comme le symptôme d'une société rendue malade ${ }^{17}$. Les idées révolutionnaires refusant l'ordre ancien, telle une épidémie, gagnaient toutes les classes sociales au début du vingtième siècle :

«Et comme des millions de personnes à cette époque, elle avait découvert qu'un ordre des choses se fissurait, près de s'écrouler, ou qu'en fait il n'y avait pas d'ordre, aucune rectitude, juste l'habitude servile qui les attachait, comme la planche dans le dos, aux lois dites naturelles...(...) Un autre poète prétendait boire le champagne 
dans le crâne de sa bien-aimée suicidée. Puis, ce mécène qui avait commandé une icône représentant un énorme succube nu... » (p.206) le comportement dénaturé du fils était déjà en germe dans les réactions contre l'ordre ancien, «naturel» que représentait le tsarisme. Il suffit d'inverser les sexes et, l'inversion s'inscrit précisément dans cette mouvance, pour que le succube désigne le fils comme un incube. La fin du passage nuance l'axiologie dégagée initialement. L'ordre qui semblait caractériser le tsarisme était davantage fondé sur l'habitude, l'absence de remise en question, une certaine acceptation des faits, qu'une véritable justesse. Néanmoins, le fait de lier l'inceste du fils à la révolution bolchevique par le recours à l'image du succube, diabolise ce régime. Le caractère allégorique des relations incestueuses entre olga et l'adolescent étant établi, le récit développe alors une conception de l'Histoire que les compétences idéologiques du lecteur feront émerger des méditations poétiques d'olga qui alternent avec les visites nocturnes que lui rend son fils : le passé porte en germe le présent ${ }^{18}$. La dernière partie du roman repose sur une attente du lecteur, vaine cette fois : l'explication tant désirée entre olga et le jeune homme n'aura pas lieu. La communication entre les deux protagonistes se limite à quelques phrases anodines avant que l'histoire, rejoignant l'Histoire, ne les sépare définitivement : le père et l'adolescent, tout comme Li, retournent en U.R.S.S., Staline ayant promis le pardon pour les émigrés repentis. A leur arrivée, les deux adultes sont envoyés dans un camp en Sibérie tandis que le sort du jeune homme demeure un mystère. Les panneaux peints de Li, dont les personnages de Psyché et Cupidon désignaient au lecteur les visites nocturnes du garçon à sa mère, sont abandonnés dans un champ neigeux derrière la gare et serviront de bois de chauffage aux paysans russes $\mathrm{du}$ coin. Dernière métaphore de la désillusion communiste après l'anesthésie du jugement que symbolisaient l'auto-aveuglement d'olga refusant la réalité puis le sommeil qu'elle mimait pour laisser son fils donner libre cours à ses désirs tératologiques. En effet, François Furet rappelle que pendant la grande famine ukrainienne de 1932 organisée par le régime (terreur multiforme exercée sur les paysans, véritable génocide qui causa cinq millions de morts), l'U.R.S.S. manipulait adroitement les personnalités étrangères en délégation officielle venues pour se rendre compte, de visu, de la situation ukrainienne. Voilà ce que dit, en 1932, Herriot, le vieux chef radical français, témoin complaisant, à son retour :

«J'ai traversé l'Ukraine. Eh bien! Je vous affirme que je l'ai vue tel un jardin en plein rendement. On assure, me dites-vous, que cette contrée vit à cette heure une époque attristée ? Je ne peux parler de ce que je n'ai pas vu. Pourtant, je me suis fait conduire dans des endroits éprouvés. Or, je n'ai constaté que la prospéritée ${ }^{19} . .$. »

Il s'est, bien sûr, laissé aveugler. Les Russes sont depuis des siècles passés maîtres dans l'art du «village Potemkine» et ils lui ont mis sous les yeux un coin d'Ukraine sur mesure. Pour expliquer cette technique illusionniste, Furet ${ }^{20}$ remonte au prince Grigori Potemkine (1739-1791), qui avait annexé la Crimée en 1783. Il organisa le voyage de Catherine II en 1787, faisant bâtir des villages fictifs peuplés de figurants déguisés en paysans pour faire croire qu'il avait conquis une province fertile et riche. Le marquis de Custine, assistant à une fête donnée au Palais d'Hiver de Saint-Pétersbourg, écrit :

«Dans les pays despotiques tous les divertissements du peuple me paraissent suspects quand ils concourent à ceux du prince. Je ne puis oublier le voyage en Crimée de l'impératrice Catherine et les façades de villages figurées de distance en distance en planches et en toiles peintes, à un quart de lieue de la route, pour faire croire à la souveraine triomphante que le désert s'était peuplé sous son règne. ${ }^{21}$ » 
prêt à succomber à l'effet-personnage, mais non pas le lectant qui s'est livré à un véritable travail de déchiffrage structurel, ni même le lu que le fantasme de l'inceste a pu combler. Le roman tout entier est construit sur cet état d'attente qui fait appel à la collaboration du lecteur dont il teste les capacités d'anticipation et aussi la patience tant les procédés dilatoires diffèrent sans cesse ce qu'il désire lire. Le dénouement, dans ce jeu avec le désir du lecteur, est exemplaire par la surprise qu'il ménage. De fait, si, durant tout le roman, la réponse attendue est toujours formulée dans l'énoncé, l'ultime réponse relative à l'identité du narrataire, apportée in extremis dans l'explicit, met en jeu l'énonciation et non plus l'énoncé.

41 C'est une véritable dialectique qu'élabore ce roman qui fait se croiser les désirs de l'auteur, du lecteur et des personnages: l'auteur inscrit le désir du lecteur dans la fabula par le truchement d'un personnage dont les pensées et les réactions représentent celles du lecteur ${ }^{22}$. Ce dernier a l'illusion que les attentes d'olga reflètent les siennes alors qu'en réalité, elles les créent. L'attente et le désir dans le texte ne réverbèrent pas l'attente et le désir du lecteur pour le texte; ils les déterminent. Le personnage d'olga constitue moins un miroir du lecteur qu'un moyen de susciter et de circonscrire ses désirs. L'instance lectorale est appelée à accomplir coopérativement les mêmes opérations de reconnaissance de relations qu'Olga, en tant que personnage, est invitée à accomplir et qu'elle exécute avec quelque retard. Ce redoublement attire l'attention du lecteur sur sa propre activité. Le lecteur est aspiré dans le texte et cet élément de jeu suscite sa participation pour résoudre le problème de lecture posé par l'œuvre. Ce roman, en racontant l'histoire d'olga (ses soupçons, ses interrogations, ses hypothèses, ses découvertes), fournit en même temps des instructions sémanticopragmatiques au lecteur dont il raconte l'histoire en le confrontant à une palette d'indices, de pistes, de leurres, de bifurcations, d'embrayages, en somme à des arborescences réactivées par un retour aux sèmes du titre dispersés plus ou moins ostensiblement dans le texte. Il lui semble que son parcours devance celui d'olga qui permet de valider ses prévisions. La structure de l'œuvre prévoit certaines réponses du lecteur de telle façon que ses réactions entrent dans les calculs de l'auteur comme si ce dernier programmait ces réponses dans le roman. Si le lecteur croit pouvoir jouer avec le texte, notamment en enquêtant, c'est d'abord parce que le texte joue avec lui. Le texte narratif est construit pragmatiquement comme la pièce de Sophocle $\mathbb{E}$ dipe Roi, qui en constitue, d'ailleurs, l'intertexte principal ${ }^{23}$. Ce qui est trouvé à la fin, c'est à la fois l'autre et soi-même. Cela vaut pour le lecteur comme pour l'auteur dont la figure surgit par le truchement de la métalepse finale qui brouille les frontières entre le narrataire, le narrateur et la figure auctoriale après que celles entre le personnage et le lecteur ont quelquefois vacillé. 


\section{NOTES}

1. Paris, Mercure de France, collection Folio, 1998.

2. Lire, juillet-août 2000, interview de Pascale Frey.

3.R. Barthes, Le plaisir du texte, Paris, Seuil, coll. « Points », 1973, p.25-26.

4.V. Jouve, L'effet-personnage dans le roman, Paris, PUF, coll. Ecriture, 1998, p.19.

5.Ibid., p.21.

6.A. Tassel, Réverbérations du titre dans « Education européenne », ROMAN 20/50, n³2, décembre 2001.

7. Ibid., p.20.

8. Ibid., p.16.

9.U. Eco, Lector in fabula, Paris, Le Livre de Poche, Biblio, Essais, Grasset, [1979] pour la traduction française, 1985, p.114.

10. Selon U. Eco, « le topic est une hypothèse dépendant de l'initiative du lecteur qui la formule d'une façon quelque peu rudimentaire, sous forme de question (« Mais de quoi diable parle-t-on?») qui se traduit par la proposition d'un titre provisoire (" On n'est probablement en train de parler de telle chose »). Il est donc un instrument métatextuel que le texte peut aussi bien présupposer que contenir explicitement sous forme de marqueurs de topic, de titres, de sous-titres, de mots clefs. », op. cit., p.116.

11. U. Eco, op. cit., p.144.

12. V. Jouve définit le lu comme une instance du lecteur qui satisfait ses pulsions inconscientes au cours de la lecture. Cf. L'effet-personnage dans le roman, Paris, PUF, coll. Ecriture, 1998, pp.89-90.

13. M. Picard, La lecture comme jeu, Paris, Les Editions de Minuit, 1986, pp.93-94.

14. P. Gutton, Le jeu chez l'enfant-Essai psychanalytique, Paris, Larousse-Université, 1973, p.53.

15. P. Pascal, Mon journal de Russie, 1918-1921, t. II, Paris, L'Age d'homme, 1977.

16. F. Furet, Le passé d'une illusion, Essai sur l'idée communiste au XXe siècle, Editions Robert Laffont, Paris, 1995, p.179.

17. Rappelons que l'un des premiers ouvrages de Lénine traduit en français est $L a$ Maladie infantile du communisme.

18. En ce qui concerne l'inscription de l'Histoire dans le roman, on peut se reporter à la quatrième partie de notre thèse : « Désir et représentation dans l'œuvre d'Andreï Makine », Université de Nice-Sophia Antipolis.

19. Fred Kupferman, Au pays des Soviets, le voyage français en Union soviétique, 1917-1939, Gallimard-Julliard, 1979, p.87-90.

20. F. Furet, op. cit., p.247.

21. Custine, Lettres de Russie, préface de P. Nora, Gallimard, Folio, 1975, pp.142-143.

22. Rappelons que Maurice Couturier définit le roman comme « une interface entre deux sujets réels, l'auteur et le lecteur, tous les deux aux prises avec leurs désirs ", in Roman et censure ou la mauvaise foi d'Eros, Paris, Champ Vallon, 1996, p.17.

23. Concernant l'intertexte oedipien, nous renvoyons le lecteur à la troisième partie de notre thèse, op. cit. 


\section{RÉSUMÉS}

Sollicitant une intense activité lectorale, Le crime d'olga Arbélina d'Andreï Makine repose sur un dispositif textuel complexe qui programme efficacement le désir de son lecteur par un ensemble de procédés convergents. Ces procédés programmatifs sont de deux sortes. En premier lieu, le titre de l'œuvre essaimé plus ou moins fidèlement dans le texte, métaphorisé, voire invalidé, convie le lecteur à une enquête dans une œuvre qui s'apparente à un roman policier. D'autre part, la représentation du désir du personnage-titre, Olga, préfigure celui du lecteur et lui désigne l'attitude à adopter, tout en entretenant l'illusion que le personnage est constamment en retard sur ses attentes. Le parcours de ce dernier balise celui du lecteur qui a pourtant l'impression que le comportement du personnage reflète, en différé, le sien. C'est moins une mise en abyme du désir métadiégétique du lecteur qu'un dispositif destiné à le susciter. La représentation du désir intradiégétique est, en effet, programmative plutôt que reproductrice. Le jeu dialectique qu'élabore le roman fait se croiser les désirs de l'auteur, du lecteur et des personnages: l'auteur inscrit le désir du lecteur dans la fabula par le truchement d'un personnage dont les pensées et les réactions représentent et déterminent celles du lecteur.

\section{INDEX}

Index chronologique : XXe siècle

\section{AUTEUR \\ EMMANUELLE OCCELLI}

Université de Nice, CNA 